\title{
Disentanglement of a Singlet Spin State in a Coincidence Stern-Gerlach Device
}

\author{
Per-Olof Westlund ${ }^{1}$, Håkan Wennerstrôm ${ }^{2}$ \\ ${ }^{1}$ Department of Chemistry, Theoretical Chemistry, Umeå University, Umeå, Sweden \\ ${ }^{2}$ Department of Physical Chemistry, Lund University, Lund, Sweden \\ Email: per-olof.westlund@umu.se
}

How to cite this paper: Westlund, P.-O. and Wennerstrôm, H. (2019) Disentanglement of a Singlet Spin State in a Coincidence SternGerlach Device. Journal of Modern Physics, 10, $1247-1254$.

https://doi.org/10.4236/jmp.2019.1010083

Received: May 8, 2019

Accepted: September 20, 2019

Published: September 23, 2019

Copyright (C) 2019 by author(s) and Scientific Research Publishing Inc.

This work is licensed under the Creative Commons Attribution International License (CC BY 4.0).

http://creativecommons.org/licenses/by/4.0/

Open Access

\section{Abstract}

We analyze the spin coincidence experiment considered by Bell in the derivation of Bells theorem. We solve the equation of motion for the spin system with a spin Hamiltonian, $H_{z}$, where the magnetic field is only in the $z$-direction. For the specific case of the coincidence experiment where the two magnets have the same orientation the Hamiltonian $H_{z}$ commutes with the total spin $I_{z}$, which thus emerges as a constant of the motion. Bells argument is then that an observation of spin up at one magnet A necessarily implies spin down at the other B. For an isolated spin system A-B with classical translational degrees of freedom and an initial spin singlet state there is no force on the spin particles A and B. The spins are fully entangled but none of the spin particles A or B are deflected by the Stern-Gerlach magnets. This result is not compatible with Bells assumption that spin 1/2 particles are deflected in a Stern-Gerlach device. Assuming a more realistic Hamiltonian $H_{z}+H_{x}$ including a gradient in $x$ direction the total $I_{z}$ is not conserved and fully entanglement is not expected in this case. The conclusion is that Bells theorem is not applicable to spin coincidence measurement originally discussed by Bell.

\section{Keywords}

Bells Theorem, Disentanglement, Stern-Gerlach Coincident Measurement, Singlet Spin State

\section{Introduction}

In 1966, Bell [1] presented an analysis of a thought experiment based on independent measurements of the spin state of two initially correlated particles. Using an example advocated by Bohm and Aharonov [2] he described the experiment as follows:

Consider a pair of spin-one-half particles formed somehow in the singlet spin state and moving freely in opposite direc- 
tions. Measurements can be made, say by Stern-Gerlach magnets, on selected components of the spins $\sigma_{\mathbf{A}}$ and $\sigma_{\mathbf{B}}$. If measurement of component $\sigma_{\mathbf{A}} \cdot \vec{a}$ where $\vec{a}$ is some $\mathrm{u}$ nit vector, yield the value +1 then, according to quantum mechanics, measurements of $\sigma_{\mathbf{B}} \cdot \vec{a}$ must yield the value -1 and vice versa. Now we make the hypothesis, and it seems one at least worth considering, that if the two measurements are made at places remote from one another the orientation of the magnet does not influence the result obtained with the other. Since we can predict in advance the result of measuring any chosen component of $\sigma_{\mathbf{B}}$, by previously measuring the same component of $\sigma_{\mathbf{A}}$ it follows that the result of any such a measurement must actually be predetermined [1].

In the present paper, we aim at scrutinizing the arguments used by Bell using recent advances [3] in the formal description of the Stern-Gerlach experiment [4]. A crucial question from our perspective, concerns the anticipated correlation between the observations in the two magnets. Bell's analysis is based on the assumption that the initial spin singlet state is preserved during the passage through the two Stern-Gerlach devises. We argue below that there is a coupling between the spin and translational degrees of freedom caused by inhomogeneities in the magnet field. This coupling leads to changes in the spin state and a partial disentanglement of the initial singlet state.

\section{The Stern-Gerlach Setup and Its Application to Coincidence Measurements}

The essential ingredients in a Stern-Gerlach experiment is i) a source of spin particles, for simplicity we consider $I=1 / 2$ particles. The source should give particles of as well-defined speed as possible. Through a slit system particles are selected to propagate in a narrow solid angle, chosen here to be in the $y$-direction. The particles then enter the gap of a magnet where the main component, $B_{z}$ of the field $B$ is perpendicular to the direction of propagation. The field $B$ is inhomogeneous with a gradient $\frac{d B_{z}}{d z}$ in the $z$-direction. It follows from Maxwells laws that there is also a matching gradient in some other direction; in the SG case the $x$-direction. Using such a setup Stern and Gerlach observed [4] that silver atoms, later realized to have spin $1 / 2$, were deflected an amount $\pm \Delta z$ in the gradient. Only these two alternatives were observed and the degree of deflection was later found to be consistent with estimates of the magnetic moment associated with the spin of an electron. The, empirical, conclusion was that the silver atoms passed through the magnet as if they were either in the $I_{z}=+1 / 2$ or $I_{z}=-1 / 2$ state, with equal probability. In the conventional, textbook analysis of the experiment the translational motion is described classically and one leaves the question open of how a spin of initially unspecified polarization appears as polarized along the direction of the magnetic field [5]. By analyzing how the predicted outcome of the experiment depended on the relative orientation of the two magnets Bell found that the expected observation at one magnet depended on the orientation of the second magnet in spite of the fact that there was no direct physical interaction between the 
particles once they had left the source. This observation, later coined Bells theorem, has given rise to a large discussion concerning nonlocality effects in quantum systems. [6] In later years the discussion of this effect has mainly been concentrated on photon systems. The present paper is solely concerned with the discussion of the original coincidence spin measurement argument.

Consider the special case when the two magnets have the same orientation in the $x z$-plane chosen to be the $z$ direction. According to the original SG observation the spin particle reaches the detector of magnet $\mathrm{A}$ at say position $+\Delta z$ indicating that $I_{z A}=+1 / 2$. What is the expected observation for the other particle of the pair at the second magnet? Following Bohms reasoning Bell concluded that by necessity $I_{z B}=-1 / 2$ for this particle and it then should be observed at $-\Delta z$ in the second magnet. To see the basis for this conclusion considered first the spin Hamiltionian

$$
\begin{aligned}
H_{z} \equiv H_{z A}+H_{z B} & =\left[-\gamma \hbar\left[B_{z}+\frac{d B_{z}}{d z} z(t)\right] I_{z}\right. \\
& +\left[-\gamma \hbar\left[B_{z}+\frac{d B_{z}}{d z} z(t)\right] I_{z} .\right.
\end{aligned}
$$

where $\gamma$ and $\hbar$ have their usual meaning. Equation (1) is typically used to analyze the SG experiment in elementary texts. It follows that the total spin in the $z$-direction $I_{z}=I_{z A}+I_{z B}$ commutes with the Hamiltonian and $I_{z}$ is a constant of the motion. For a singlet state, $\left|S_{0}\right\rangle$, it has a value $I_{z}=0$ so $I_{z A}=1 / 2$ implies $I_{z B}=-1 / 2$, or vice versa. By necessity there is, within the magnet, also a field in the $x$-direction and thus two terms

$$
\begin{aligned}
H_{x} \equiv H_{x A}+H_{x B} & =\left[-\gamma \hbar \frac{d B_{z}}{d x} x(t) I_{x}\right]_{A} \\
& \left.+-\gamma \hbar \frac{d B_{z}}{d x} x(t) I_{x}\right]_{B}
\end{aligned}
$$

in the spin Hamiltonian. Bohm argued that the gradient in the $x$ direction was of negligible consequence $[7,8]$ so that it is a good approximation to analyze the experiment on the basis of a further simplified Hamiltonian $H_{z}$. Still $I_{z}$ is strictly not a constant of the motion.

The analysis of the coincidence experiment by Bell is based on two basic arguments. The first is the empirical finding of the SG experiment that a spin $1 / 2$ particle is deflected an amount $\pm \Delta z$. The second is that for parallel orientations of the two magnets the total spin $I_{z}$ is conserved which follows theoretically using the Hamiltonian $H_{z}$. The dependence of the outcome on the relative orientation of the two magnets then follows in a straightforward way. We note that the two basic arguments have different origins; one empirical and one theoretical.

\section{Describing the Stern-Gerlach Experiment through the Hamiltonian $H_{z}$}

It is implicit in the Hamiltoinan $H_{z}$ that the translational degrees of freedom are described classically. We now investigate to which extent this Hamiltonian describes the dynamics of spin particles in the SG magnet. In contrast to the original SG experiment the initial spin state is known and represented by an entangled singlet in the coincidence setup. The equation of motion for the spin "density operator", $\sigma(t)$, 
is

$$
\frac{d}{d t} \sigma(t)=-\frac{i}{\hbar}\left[H_{z}, \sigma(t)\right]
$$

To illustrate our point it is sufficient to consider the special case with only one Stern-Gerlach device. Then

$$
H_{z}=H_{z A}
$$

For two $\mathrm{I}=1 / 2$ particles there are 16 spin density operator components. For the case with a magnetic field solely along the z-direction only four components couple; $\left|S_{0}><S_{0}\right|,\left|S_{0}><T_{0}\right|, \mid T_{0}><$ $S_{0}|,| T_{0}><T_{0} \mid$. Here $\left|S_{0}\right\rangle$ denotes the singlet state and $\left|T_{0}\right\rangle$ the triplet state with $S_{z}=0$. The equation of motion,

$$
\begin{gathered}
\frac{d}{d t}\left[\begin{array}{l}
\sigma_{A B}(t)_{\left|S_{0}><S_{0}\right|} \\
\sigma_{A B}(t)_{\left|S_{0}><T_{0}\right|} \\
\sigma_{A B}(t)_{\left|T_{0}><S_{0}\right|} \\
\sigma_{A B}(t)_{\left|T_{0}><T_{0}\right|}
\end{array}\right]= \\
\frac{1}{2}\left[\begin{array}{lllr}
0 & -i \omega_{A}(z) & i \omega_{A}(z) & 0 \\
-i \omega_{A}(z) & 0 & 0 & i \omega_{A}(z) \\
i \omega_{A}(z) & 0 & 0 & -i \omega_{A}(z) \\
0 & i \omega_{A}(z) & -i \omega_{A}(z) & 0
\end{array}\right]\left[\begin{array}{l}
\sigma_{A B}(t)_{\left|S_{0}><S_{0}\right|} \\
\sigma_{A B}(t)_{\left|S_{0}><T_{0}\right|} \\
\sigma_{A B}(t)_{\left|T_{0}><S_{0}\right|} \\
\sigma_{A B}(t)_{\left|T_{0}><T_{0}\right|}
\end{array}\right]
\end{gathered}
$$

where $\omega_{A}(z) \equiv-\gamma_{A}\left[B_{z}^{A}+\frac{d B_{z}^{A}}{d z_{A}} z_{A}\right]$. With the initial condition $\rho(0)_{\left|S_{0}><S_{0}\right|}=1$, the solution is (see SI)

$$
\begin{aligned}
& \sigma_{A B}(t)_{\left|S_{0}><S_{0}\right|}=\frac{1}{2}\left(1+\cos \left(\omega_{A}(z) t\right)\right. \\
& \sigma_{A B}(t)_{\left|S_{0}><T_{0}\right|}=-\frac{1}{2}\left(i * \sin \left(\omega_{A}(z) t\right)\right. \\
& \sigma_{A B}(t)_{\left|T_{0}><S_{0}\right|}=\frac{1}{2}\left(i * \sin \left(\omega_{A}(z) t\right)\right. \\
& \sigma_{A B}(t)_{\left|T_{0}><T_{0}\right|}=\frac{1}{2}\left(1-\cos \left(\omega_{A}(z) t\right)\right.
\end{aligned}
$$

The force, $F_{A}$, on the particle $\mathrm{A}$ is

$$
F_{A}=\frac{d B_{z}}{d z_{A}}\left\langle I_{z, A}\right\rangle(t)=0
$$

since

$$
\left\langle I_{z, A}\right\rangle(t)=\frac{1}{2}\left[\operatorname{tr}_{B}\left\{\sigma_{A B}(t)_{\left|S_{0}><T_{0}\right|}\right\}+\operatorname{tr}_{B}\left\{\sigma_{A B}(t)_{\left|T_{0}><S_{0}\right|}\right\}\right]=0
$$

It follows that there is no net force on the particles and the Hamiltonian $H_{z A}\left(H_{z}\right)$ is thus inadequate for predicting the empirically based anticipated outcome of the experiment. On the other hand the calculation show that the two separated spins A and B are completely entangled. This indicates that there is an internal inconsistency in Bell's original argument. The Hamiltonian used to motivate that $I_{z}$ is a constant of motion is inadequate to explain why one observe a spin dependent deflection of particles emerging from a non-magnetic singlet spin state.

\section{Implications of Recent Description of the SG Experiment}

There has in recent years appeared several theoretical accounts of the SG experiment [9]- [14]. Although clearly different in the approaches 
they share the common feature that it is necessary to include a quantum description of the translational motion or other degrees of freedom to account for the observation of two distinct positions $\pm \triangle z$ at the detector for a spin $1 / 2$ particle. This further corroborates the conclusion of the previous section that the Hamiltonian $H_{z}$ of Equation (1) is inadequate for describing the SG-experiment. A more adequate Hamiltoinan $[13,14]$ for describing the experiment can be written

$$
H=\vec{p} / 2 m+\gamma \vec{S} \cdot \vec{B}(\hat{r})
$$

where $B(\hat{r})$ is the magnetic field and the hat $(\hat{r})$ is to denote that the spatial coordinate is an operator in the Hamiltonian. In most discussions of the SG experiment the focus is on the magnetic field inside the magnet, neglecting the gradient in the $\mathrm{x}$-direction so that

$$
B=B_{z}+\frac{d B_{z}}{d z} \hat{z}
$$

Based on this expression for the magnetic field the outcome of the S$\mathrm{G}$ experiment is adequately predicted. Using a perturbation approach we [3] could also show that the effect of the gradient in the $x$-direction is to reduce the deviation for spin particles with initial positions slightly off center. This calculation corroborates Bohms original assertion that the $x$-gradient has only a small influence. However, it is causing the mouth-like shape found at the detector in the original experiment. When applied to the coincidence measurement the Hamiltonian (9) with the expression for the magnetic field (10), for the respective particles, gives a system where the total spin $I_{z}$ is conserved. However, there is an important factor missing in the expression for the magnetic field in Equation (10), which refers to the conditions inside the magnet. There is necessarily a zone close to the entrance of the gap of width $\mathrm{D}$ in the magnet where the $z$-component of the field grows from zero to $B_{z}$. From Maxwells equations it follows that there are also a gradient of corresponding magnitude in the $y$-direction, as well as a small contribution from the asymmetry in the $x$-direction. In [3] we have presented an approximate analytical expression for the field outside the magnet, $y \leq 0$.

$$
\begin{aligned}
B_{z}(y, z) & \approx \frac{B_{0}}{\pi}\left\{\arctan \left(\frac{z+D}{y}\right)-\arctan \left(\frac{z-D}{y}\right),\right. \\
B_{y}(y, z) & \approx-\frac{B_{0}}{2 \pi} \ln \left\{\frac{y^{2}+(z+D)^{2}}{y^{2}+(z-D)^{2}},\right\} \\
B_{x} & \approx 0 .
\end{aligned}
$$

Using the expression (11) for the field in the Hamiltonian (9) it follows that the commutator becomes

$$
\left[H, I_{z}\right]=\gamma\left(I_{x A} B_{y A}(\hat{y}, \hat{z})+I_{x B} B_{y B}(\hat{y}, \hat{z})\right) .
$$

This shows that $I_{z}$ is not a constant of the motion since the commutator of Equation (12) is not zero. To further emphasize the existence of a coupling between spin and translation we note that there is an effect on the momentum in the $y$-direction in the entrance zone. This is evident from the nonzero value of the commutator $\left[H, p_{y}\right]$, caused by the $y$-dependence of both $B_{z}$ and $B_{y}$ in Equation (11). In the entrance phase there is thus, in general, an energy transfer between spin and translation degrees of freedom. 
It is a considerable challenge to solve the equation of motion for the combined spin-translation degrees of freedom. Lacking such a solution we consider possible implications of the coupling between spin and translation by analyzing how the spin energy levels vary as the initially strongly coupled spins separate and move towards their respective magnets. For the sake of illustration let the two magnets be positioned as symmetrically as possible. As the two spin particles move apart they still interact through a dipolar coupling, while the stronger J-coupling is vary local. The dipolar coupling results in two non-degenerate states, $\left|S_{0}\right\rangle$ and $\left|T_{0}\right\rangle$, both with $I_{z}=0$, but with different permutation symmetry. In addition there is a degenerate level with states $\left|T_{1}\right\rangle$ and $\left|T_{-1}\right\rangle$. As the particles separate the dipolar coupling becomes negligible while there is a growing Zeeman term from the magnetic field. In this limit, and assuming the z-component of the field to be the largest one, the $\left|T_{1}\right\rangle$ and $\left|T_{-1}\right\rangle$ levels are separated by a frequency $2 \omega(y)$, while the $\left|S_{0}\right\rangle$ and $\left|T_{0}\right\rangle$ states are degenerate with an energy half way between the other levels (See Figure 1). With a perfect symmetry between the two magnets an initial singlet state will remain in that state throughout the passage. However, it is not feasible to have microscopically identical fields in the two magnets. A difference in the $z$-components of the magnetic fields will result in a mixing of the $\left|S_{0}\right\rangle$ and $\left|T_{0}\right\rangle$ states, while the $I_{z}$ component is conserved at a value zero. A difference in the $B_{x}$ or $B_{y}$ components result in a breaking of the symmetry with respect to both parity and $I_{z}$. In Figure 1 it is seen that the energy level for the singlet state by necessity crosses either the level for the $\left|T_{-1}\right\rangle$ state, as in the figure, or for the $\left|T_{1}\right\rangle$ state, for a different choice of the sign of the gyromagnetic ratio.

One approach to handle the problem of treating the quantum effects of the translational degree of freedom is to use adiabatic approximation. In the specific example of Figure 1 the adiabatic reasoning would predict that the initial $\left|S_{0}\right\rangle$ state will experience an avoided crossing in the presence of a coupling term and continue in the $\left|T_{1}\right\rangle$ state as the particles enter the magnet. As expected neither the parity nor the $I_{z}$ value is conserved during the crossover. This argument shows, by example, a realistic mechanism for how the $I_{z}$ value of the initial singlet state can change during the entrance of the magnet, by referring to the common phenomenon of avoided level crossing .

\section{Conclusion}

We have analyzed the arguments leading to the formulation of Bells theorem for spin coincidence measurements. The proof of the theorem has two central ingredients. The rule, based on the original SternGerlach observation, that on exit from the SG-setup a spin $1 / 2$ particle is either in the $I_{z}=+1 / 2$ or $I_{z}=-1 / 2$ state. The second essential feature is that the total spin in the $z$-direction $I_{z}$ is conserved. This conservation rule follows from the Hamiltonian Equation (1) usually used in simplified discussions of the SG experiment. However, using the Hamiltonian of Equation (1) and the known initial spin state the equation of motion for the spin can be solved and we find that there is no net force acting on the particle passing through the magnet, as expected for a non-magnetic state. This indicates that there is an internal inconsistency of the original proof of Bells theorem. Recent work [3], [10]- [12] has shown that it is necessary to include a quantum description of the translational motion or other degrees of freedom in order to account for the observed SG effect. The empirical rule used 


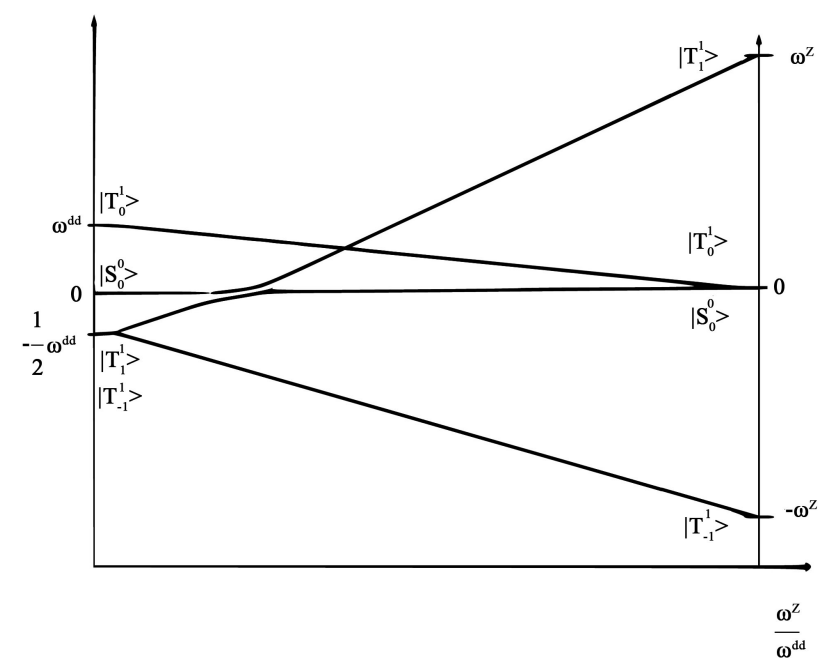

Figure 1. The energy level at zero magnetic field with the eigenstates $\left|S_{0}^{0}\right\rangle,\left|T_{0}^{1}\right\rangle,\left|T_{1}^{1}\right\rangle$ and $\left|T_{-1}^{1}\right\rangle$ with the dipole-dipole energy $\omega^{d d}$. Increasing Zeeman energy $\omega^{Z}$ to the right, indicating how the energy leves change for the states when $\omega^{Z} \gg \omega^{d d}$.

by Bell can thus be theoretically derived, but using a different Hamiltonian than Bell used to conclude that $I_{z}$ of the combined system is conserved. The coupling between spin and translation emerges from the spatial dependence of the magnetic field, which has components in more than one direction. It follows that the $I_{z}$ of the combined system is not a constant of the motion. We further argue that the most significant deviation of the field from the main $z$-direction occurs on entering the magnet. The proof of Bells theorem thus rests on an approximation of unknown validity. We have given one illustration of the situation where using conventional argument, where the approximation is specifically giving a misleading result.

\section{Acknowledgements}

This work was supported by Swedish Research Council (VR).

\section{Conflicts of Interest}

The authors declare no conflict of interest.

\section{References}

[1] Bell, J.S. (1966) Reviews of Modern Physics, 38, 447-452.

[2] Bohm, D. and Aharonov, Y. (1957) Physical Review, 108, 1070. https://doi.org/10.1103/PhysRev.108.1070

[3] Wennerstrom, H. and Westlund, P.-O. (2017) Entropy, 19, 186. https://doi.org/10.3390/e19050186

[4] Gerlach, W. and Stern, O. (1922) Zeitschrift fr Physik, 9, 349352. https://doi.org/10.1007/BF01326983

[5] Einstein, A. and Ehrenfest, P. (1922) Zeitschrift fr Physik, 11, 31-34. https://doi.org/10.1007/BF01328398 
[6] Schlosshauer, M., Ed. (2011) Elegance and Enigma. Springer Heidelberg, Dordrecht, London, New York.

[7] Bohm, D. (1952) Physical Review, 85, 180. https://doi.org/10.1103/PhysRev.85.180

[8] Bohm, D. (1951) Quantum Theory. Dover Publications, New York, 326 .

[9] Scully, M.O., Shea, R. and McCullen, J. (1978) Physics Reports, 43, 485-498. https://doi.org/10.1016/0370-1573(78)90210-7

[10] Scully, M.O., Lamb Jr., W.E. and Barut, A. (1987) Foundations of Physics, 17, 575-583. https://doi.org/10.1007/BF01882788

[11] Wennerstrom, H. and Westlund, P.-O. (2012) Physical Chemistry Chemical Physics, 14, 1677-1684. https://doi.org/10.1039/C2CP22173J

[12] Wennerstromm H. and Westlund, P.-O. (2013) Physics Essays, 26, 174-180. https://doi.org/10.4006/0836-1398-26.2.174

[13] Utz, M., Levitt, M.H., Cooper, N. and Ulbricht, H. (2015) Physical Chemistry Chemical Physics, 17, 3867-3872. https://doi.org/10.1039/C4CP05606J

[14] Gomis, P. and Perez, A. (2016) Physical Review A, 94, Article ID: 012103. https://doi.org/10.1103/PhysRevA.94.012103 